\title{
DRIZZLE MEASUREMENTS USING HIGH SPECTRAL RESOLUTION LIDAR AND RADAR DATA
}

\author{
Edwin W. Eloranta
}

University of Wisconsin, Madison, WI 53706, USA, *Email: eloranta@ssec.wisc.edu

\begin{abstract}
The ratio of millimeter radar and High Spectral Resolution Lidar (HSRL) backscatter are used to determine drizzle rates which are compared to conventional ground based measurements. The robustly calibrated HSRL backscatter cross section provides advantages over measurements made with traditional lidars.
\end{abstract}

\section{INTRODUCTION}

Marine stratus clouds are an important feature of the global climate system. Cloud lifetime is sensitive to drizzle rates. Drizzle not only removes water from the cloud but evaporation of the falling droplets cools the sub-cloud layer acting to suppress convection. Accurate measurements of drizzle rates will improve our understanding of cloud maintenance.

Several investigators have used simultaneous lidar and radar observations to determine particle size $[1,2,3]$. However, measurements made with conventional lidar are hampered by: 1) changes in the transmission of the output window caused by water accumulation, 2) the difficulty of correcting the backscatter signal for atmospheric extinction, 3 ) the effects of multiple scattering, and 4) the need to convert backscatter measurements to extinction. The use of High Spectral Resolution Lidar (HSRL) data avoids many of these problems. HSRL backscatter measurements are referenced to the known molecular scattering cross-section at each point in the profile and are thus independent of changes in window and atmospheric transmission.

This study uses data collected during the US Department of Energy Atmospheric Sciences program MAGIC campaign. Instruments including a suite of conventional precipitation gauges, a High Spectral Resolution Lidar, along with $3.2 \mathrm{~mm}$ wavelength WACR and a $8.6 \mathrm{~mm}$ wavelength KAZR radars, were installed on the container ship Horizon Spirit as it made repeated trips between Long Beach, CA and Honolulu, HI. Figures 1 though 4 provide an example of the measurements used in our retrieval of drizzle rates.

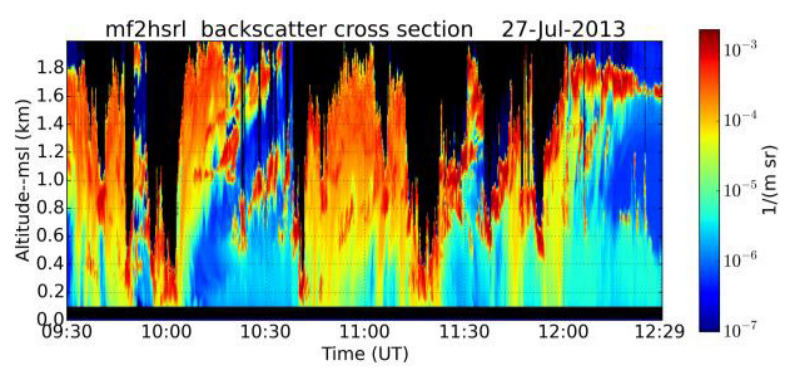

Figure 1: HSRL measured 532nm backscatter cross-section on 27-Jul-2013. Water clouds produce the areas with backscatter cross-sections near 1e-3 $1 /(m$ sr). Drizzle shafts near the surface appear with backscatter cross-sections between $1 \mathrm{e}-5$ and 1e-4 1/(m sr).

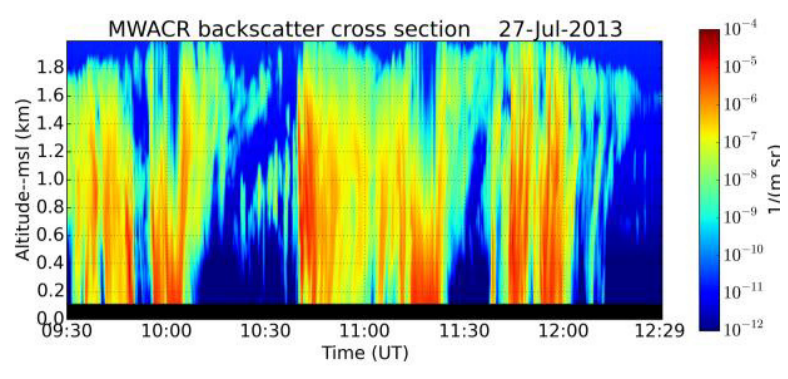

Figure 2: Radar backscatter cross section measured with the WACR $3.2 \mathrm{~mm}$ wavelength radar. (note: cross section is $4 \pi$ times usual radar definition).

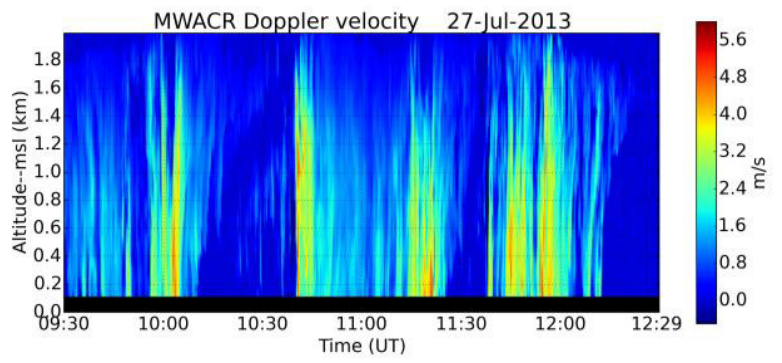

Figure 3: Doppler velocity measured with the WCAR radar on 27-Jul-2013. 


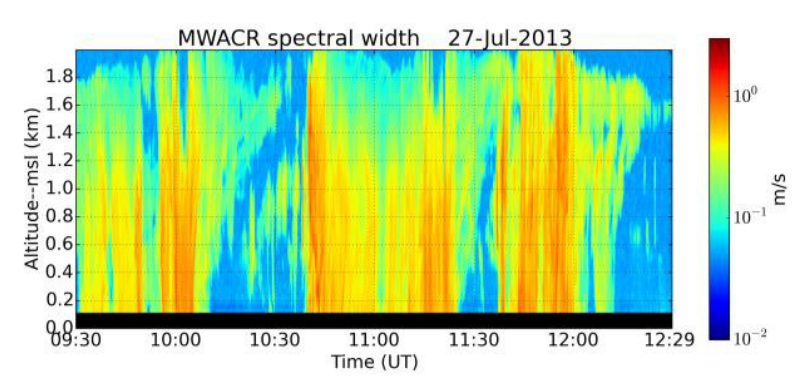

Figure 4: Spectral width measured with the WACR radar on 27-Jul-2013.

\section{DIZZLE PARTICLE SIZE RETRIEVAL}

We assume a modified gamma distribution [4] of particle diameters:

$\frac{d N}{d D} \sim D^{\alpha} \cdot \exp \left(-\frac{\alpha}{\gamma} \cdot\left(\frac{D}{D_{m}}\right)^{\gamma}\right)$

Where: $\mathrm{N}=$ number of particles

$$
\begin{aligned}
& \mathrm{D}=\text { particle diameter } \\
& \mathrm{D}_{\mathrm{m}}=\text { mode diameter } \\
& \alpha, \gamma=\text { shape parameters }
\end{aligned}
$$

Using Mie theory we compute the backscsatter cross-section as a function of size parameter for both $532 \mathrm{~nm}$ and $8.6 \mathrm{~mm}$ wavelengths using the appropriate indices of refraction. Calculations extend over the range of diameters between 1 micron and $5 \mathrm{~mm}$. The optical cross-sections were carried out at size parameter intervals of 0.01 and averaged to a spacing of 1 size parameter.

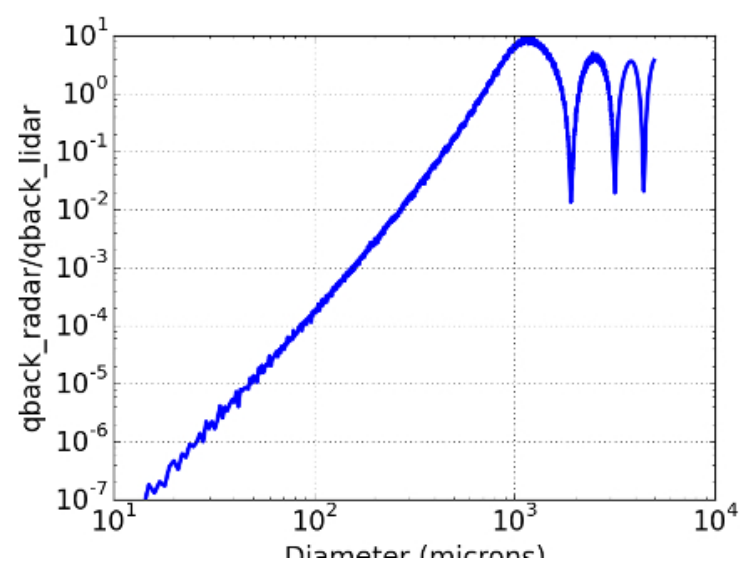

Figure 5: Ratio of $\operatorname{radar}(\lambda=3.2 \mathrm{~mm})$ to lidar $(\lambda=532$ nm) backscatter efficiencies for monodisperse particle size distribution.

It is convenient to define a diameter $\mathrm{D}^{*}$ as a ratio of the radar and lidar backscatter cross-sections:
$D *=\lambda_{\text {radar }} \cdot\left(\frac{\text { bsRadar }}{\text { bsLidar }}\right)^{\frac{1}{4}}$

This ratio can be computed for our size distribution using Mie theory backscatter efficiencies $\mathrm{Q}_{\mathrm{bsRadar}}$ for radar and $\mathrm{Q}_{\mathrm{bsLidar}}$ for lidar scattering along with the radar wavelength, $\lambda_{\text {radar }}$ :

$D *=\lambda_{\text {radar }} \cdot\left(\frac{\int D^{2} \cdot N(D) \cdot Q_{b s R a d a r} \cdot d D}{\int D^{2} \cdot N(D) \cdot Q_{b s L i d a r} \cdot d D}\right)^{\frac{1}{4}}$

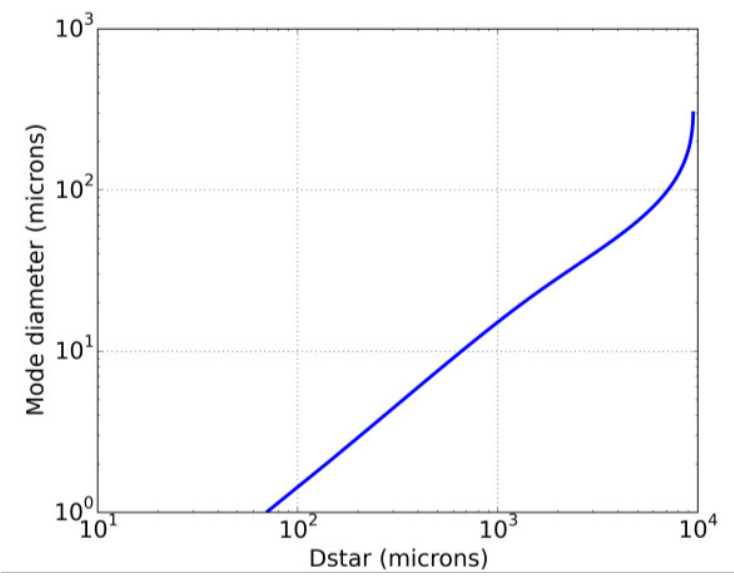

Figure 6: The relationship between $D *$ and mode diameter for a modified gamma distribution calculated with $\gamma=0.7$ and $\alpha=1$ for lidar and radar wavelengths of $532 \mathrm{~nm}$ and $3.2 \mathrm{~mm}$ respectively at a temperature of $285 \mathrm{~K}$.

This relationship allows mode diameters to be derived from $\mathrm{D}^{*}$ measurements. Formulating the relationship in terms of the backscatter crosssection takes advantage of the robustly calibrated HSRL measurement. This measurement does not require corrections for window transmission or atmospheric extinction below the scattering volume. In addition, multiple scattering effects largely cancel out in the HSRL backscatter retrieval.

Using Mie theory for the radar avoids errors caused by the Rayleigh approximation. However, the maximum mode diameter which can be retrieved is limited because the relationship between $\mathrm{D}^{*}$ and the mode diameter becomes multi-valued when the particle sizes increase into the Mie regime .

Because the size distribution is known the mode diameter can be easily converted to effective diameter (effective diameter $=$ ratio of particle volume to area). 


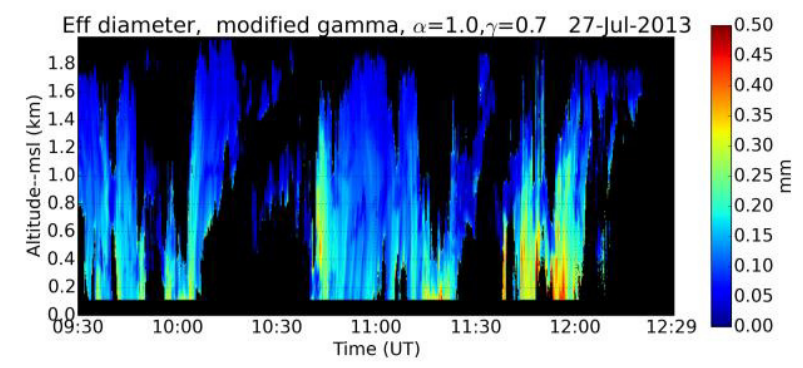

Figure 7: Effective diameter for the data shown with $\xi=1$ and $\gamma=0.7$.

Beard's parameterization [5] of fall velocity is used to compute the radar cross-section weighted fall velocity. The radar measured Doppler velocity and spectral width are independent measurements that can be compared to the modeled radar weighted velocities. Values of the shape parameters, $\alpha$ and $\gamma$ can be adjusted to improve the comparison between the measured and modeled velocities.

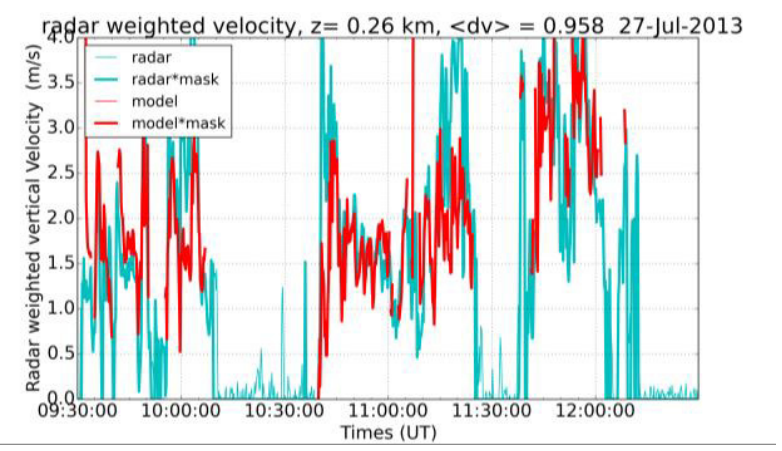

Figure 8: Comparison between measured Doppler velocities and the modeled radar weighted fall velocity at an altitude of $130 \mathrm{~m}$ with $\alpha=1, \gamma=0.7$.

Even though we do not expect exact agreement because the Doppler velocity includes contributions due to vertical air motion, figures 8 and 9 show good agreement between the retrieved radar weighted velocities and the measured Doppler velocities.

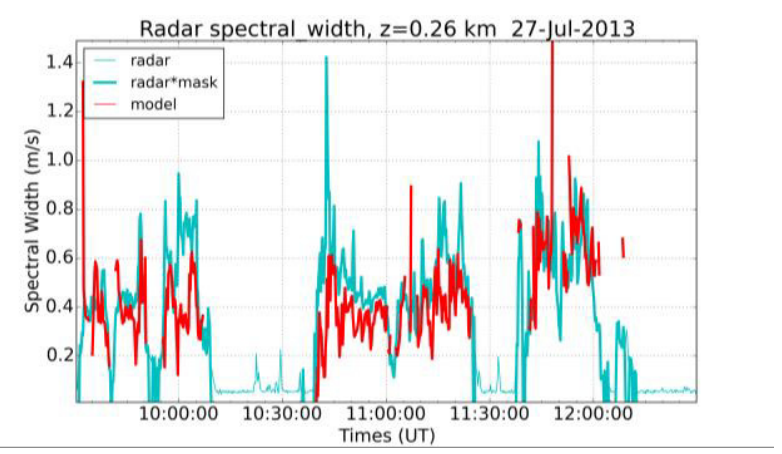

Figure 9: Comparison of measured and modeled spectral width as a function of time at an altitude of $130 \mathrm{~m}$ assuming $\alpha=1$ and $\gamma=0.7$.

\section{DRIZZLE RATE RETRIEVAL}

The extinction cross-section, of particles that are large compared to the wavelength is twice the geometric area [6]:

$$
\beta_{\text {ext }}=2 \cdot N<A>
$$

Where:

$$
\begin{aligned}
& \mathrm{N}=\text { particle number density } \\
& <\mathrm{A}>=\text { average geometric area of particles }
\end{aligned}
$$

Because the effective diameter is $\left\langle\mathrm{D}^{3}\right\rangle \mid\left\langle\mathrm{D}^{2}\right\rangle$, the liquid water content(LWC) can be computed using the density of water:

$$
L W C=\frac{1}{3} \cdot \rho_{w} \cdot D_{e f f} \cdot \beta_{e x t}
$$

The lidar extinction cross-section is derived from the lidar backscatter cross-section using:

$$
\beta_{\text {ext }}=\text { bsLidar } \cdot \frac{\int D^{2} \cdot N(D) \cdot Q_{e x t}(D) \cdot d D}{\int D^{2} \cdot N(D) \cdot Q_{b s}(D) \cdot d D}
$$

Where :

$\mathrm{Q}_{\mathrm{ext}}$ and $\mathrm{Q}_{\mathrm{bs}}$ are extinction and backscatter efficiencies calculated from Mie theory. 


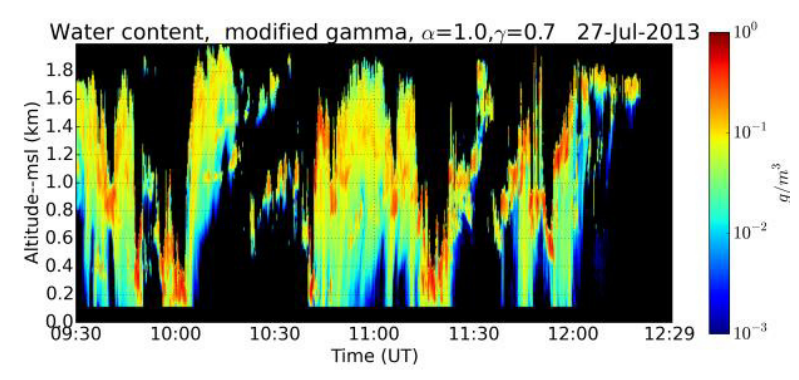

Figure 10: Liquid water content.

The derived liquid water content is not valid where clouds and drizzle are present together because this retrieval has assumed a mono-modal size distribution.

With the particles size distribution known, the mass weighted fall velocity can be computed using the Beard parameterization. The precipitation rate is computed from the product of the mass weighted fall velocity and the liquid water content.

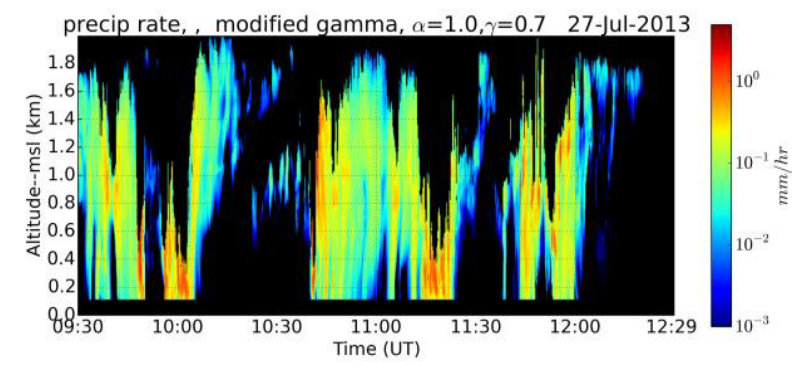

Figure 11: Precipitation rate image for 27-Jul-2013 derived using $\alpha=1, \gamma=0.7$.

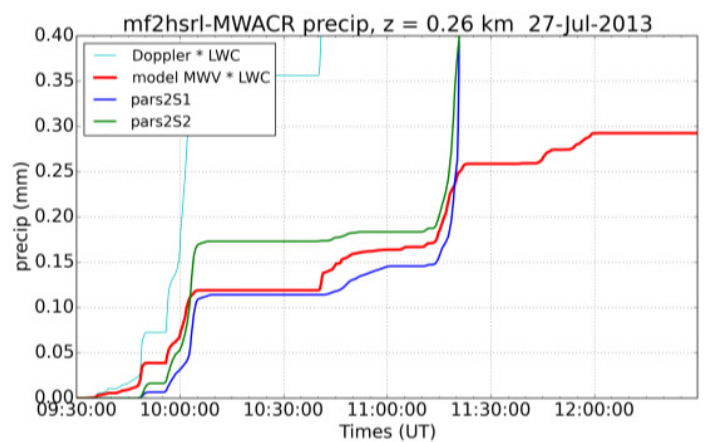

Figure 12: The accumulated precipitation measured by two shipboard distrometers (pars2S1 and pars2S2) and the lidar-radar retrieval. Also shown is the derived liquid water content multiplied by the radar Doppler velocity.

Figure 11 highlights the difficulty of making conventional precipitation measurements on a moving ship. Lidar-radar retrievals agree with PAR2S1 measurements prior to 11:45 UT, but do not agree as well with PARS2S2 measurements. After 11:45UT, the two distrometers give very different precipitation rates. Two conventional rain gauges on the ship also provide wildly different precipitation rates. Using the $8.6 \mathrm{~mm}$ KAZR radar data in the retrieval indicated $\sim 10 \%$ less precipitation with a best fit to velocities using $\gamma \sim 0.5$. This difference may be related to radar calibrations differences or because the WACR antenna was gyro-stabilized, while KZAR pointing angle rolled with the ship.

\section{CONCLUSIONS}

Lidar-Radar drizzle retrievals using HSRL data show considerable promise. The technique is sensitive to the functional form of the assumed size distribution and needs to be tested in a setting where more accurate conventional measurements are possible.

\section{ACKNOWLEDGEMENT}

This research was support by Department of Energy Grant DE-SC0006933.

\section{REFERENCES}

[1] Interi J.M., J. L. Stephens, W. L. Eberhard and, T. Uttal, 1993: A Method for Determining Cirrus Cloud Particle Sizes Using Lidar and Radar Backscatter Technique, J. Applied Meteor., 32, 1074-1082.

[2] Donovan, D.P., and A.C.A.P. van Lammeren, 2001: Cloud effective particle size and water content profile retrievals using combined lidar and radar observations: 1 . Theory and examples, $J$. Geophy. Res. Atmos., 106, 27425-27448.

[3] O'Connor, E. J., A. J. Illingworth, R. J. Hogan, 2004: Retrieving Statocumulus Drizzle Parameters Using Doppler Radar and Lidar, $J$. Atmos. Ocean Tech., 21(5), 777-778.

[4] Deirmendjain, D., 1969: Electromagnetic Scattering on Spherical Polydispersions.

American Elsevier, 290 pp.

[ 5] Beard, K. V., 1976: Terminal velocity and shape of cloud and precipitation drops aloft. $J$. Atmos. Sci., 33, 851-864.

[6] van de Hulst, H. C., 1957: Light Scattering by Small Particles. John Wiley and Sons, 470 pp. 